\title{
Numerical calculation of the wave-vector dependent Cole-Cole diagram of Copper
}

\author{
Abu El-Hassan Seoud and Mohammed Shihab \\ Physics Department, Faculty of science, Tanta University, Tanta 31527, Egypt \\ mohammed.shibab@science.tanta.edu.eg
}

\begin{abstract}
The Cole-Cole diagram of Copper is calculated in the energy range of $0 \leq \hbar \omega \leq 10$ Ryd using realistic band energies and wave-functions. Four wave-vectors $\mathrm{q}$ are chosen in $\Delta, \Lambda$, and $\Sigma$ directions, namely, $\left\langle\frac{1}{4}, 0,0\right\rangle,\left\langle\frac{1}{4}, \frac{1}{4}, 0\right\rangle,\left\langle\frac{1}{4}, \frac{1}{4}, \frac{1}{4}\right\rangle$ and $\left\langle\frac{1}{2}, 0,0\right\rangle$ in the units of $2 \pi a^{-1}$. The modified augmented-plane-wave method with the Chodorow potential is utilized to calculate the energy spectrum and Bloch wave functions. The Cole-Cole diagrams exhibit anti-clockwise rotation as the frequency increases for all $q>0$. However, the rotation direction is reversed - becomes clockwise - in the spatial case $q=0$. The static dielectric function deceases as $q^{-2}$, the area of the Cole-Cole diagram decreases as the wave-vector increases. The free electrons in Copper metal exhibits two plasmons for wave-vectors smaller than $0.52 \pi a^{-1}$. In order to show the effect of core polarization and interband transitions, the Cole-Cole diagrams of a free electron gas (Lindhard) are shown.

Keywords: Lindhard dielectric function, Wave-vector dependent Cole-Cole diagram, Copper dielectric function.
\end{abstract}

\section{Introduction}

The dielectric function describes how electromagnetic waves interact when propagating through matter. Consequently, it determines the electrical and optical properties of the material [1]. Its real part $\epsilon^{\prime}(\mathbf{q}, \omega)$ amounts the polarization and energy storage. The imaginary part $\epsilon^{\prime \prime}(\mathbf{q}, \omega)$ represents the absorption properties of the material [2]. Classical models of dielectric function such as Drude model or Drude-Lorentz model calculate very well the absorption of photons by the free electrons inside a band. However, they can't resolve band to band transitions. In order to include interband transitions, a detailed calculations of the band structure of the material is necessary and one has to invoke quantum mechanical models.

The parametric semicircular plot (Cole-Cole diagram) of the imaginary part versus the real part has been used frequently to describe the materials which obey Debye theory $[3,4]$. The dielectric function depends mainly on four parameters; the static dielectric constant $\epsilon_{0}$, the dielectric constant at infinite frequency $\epsilon_{\infty}$, the relaxation time $\tau$, and an exponent factor $\beta$ :

$$
\epsilon(\mathbf{q}, \omega)=\epsilon^{\prime}(\mathbf{q}, \omega)+i \epsilon^{\prime \prime}(\mathbf{q}, \omega)=\epsilon_{\infty}+\frac{\epsilon_{0}-\epsilon_{\infty}}{1+(i \omega \tau)^{1-\beta}}
$$

In such case the Kramers-Kronig relations can be simplified as:

$$
\epsilon^{\prime 2}-2 d \epsilon^{\prime}+\epsilon^{\prime \prime 2}=0
$$

where $d$ is the diameter of the semicircular plot.

Recently, the copper is employed as interconnect metal instead of Aluminum to achieve a higher electrical performance of on-chip wiring $[5,6,7,8]$. Therefore, we provide the calculation of the copper dielectric function. Four wave-vectors $\mathbf{q}$ are chosen in the main crystallographic directions $\Delta, \Lambda$, and $\Sigma$,namely, $\left\langle\frac{1}{4}, 0,0\right\rangle$, $\left\langle\frac{1}{4}, \frac{1}{4}, 0\right\rangle,\left\langle\frac{1}{4}, \frac{1}{4}, \frac{1}{4}\right\rangle$ and $\left\langle\frac{1}{2}, 0,0\right\rangle$ in the units of $2 \pi a^{-1}$. For more analysis, the complex dielectric function has been calculated assuming a homogeneous free electron gas for the same four wave-vectors.

\section{Dielectric function calculation}

The microscopic copper dielectric function has been calculated employing the Adler [9] definition:

$$
\epsilon(\mathbf{q}, \omega)=1-\frac{4 \pi e^{2}}{\Omega_{0} q^{2}} \alpha(\mathbf{q}, \omega)
$$

where the non-diagonal elements of the inverse dielectric matrix are fairly small [10], i.e., local field effects can be neglected. The irreducible polarization function $\alpha(\mathbf{q}, \omega)$ is given in the random phase approximation by the following sum over occupied and unoccupied Bloch states:

$$
\begin{aligned}
\alpha(\mathbf{q}, \omega) & =2 \sum_{n, n^{\prime}, \mathbf{K}}\left\langle n \mathbf{K}\left|e^{-i \mathbf{q} \cdot \mathbf{r}}\right| n^{\prime}, \mathbf{K}+\mathbf{q}\right\rangle \\
& \times\left\langle n^{\prime}, \mathbf{K}+\mathbf{q}\left|e^{i \mathbf{q} \cdot \mathbf{r}}\right| n \mathbf{K}\right\rangle \\
& \times \frac{f(n, \mathbf{K})-f\left(n^{\prime}, \mathbf{K}+\mathbf{q}\right)}{E(n, \mathbf{K})-E\left(n^{\prime}, \mathbf{K}+\mathbf{q}\right)+\hbar \omega+\mathbf{i} \eta}(4)
\end{aligned}
$$

where $\Omega_{0}$ is the crystal volume. $E(n, \mathbf{K})$ is the energy and $\langle\mathbf{r} \mid n \mathbf{K}\rangle=\psi_{n \mathbf{K}}(\mathbf{r})$ is a Bloch state wave function characterized by the wave-vector and the reciprocal lattice vector $\mathbf{K}$ and the band index $n$, and $f(n, \mathbf{K})$ is the occupation number (i.e, Fermi-Dirac distribution), $\eta$ is a positive infinitesimally small real number; $\eta \rightarrow 0^{+}$. It is worth to report that the dielectric function in eq. (3) has been derived via the perturbation of the single particle Liouville equation $i \hbar \partial \rho / \partial t=[H, \rho][9] . \quad \rho$ and $H$ denote the single-particle matrix and the Hamiltonian, respectively. Quadratic and higher orders are ignored and consequently our calculations are valid in the frame of the random phase approximation.

The matrix elements in eq. (4) are integrals over the Winger-Seitz cell (WSC):

$$
\begin{aligned}
& \left\langle n^{\prime}, \mathbf{K}+\mathbf{q}\left|e^{i(\mathbf{q}+\mathbf{K}) \cdot \mathbf{r}}\right| n \mathbf{K}\right\rangle \\
= & \frac{1}{V_{c}} \int_{W S C} \psi_{n^{\prime}, \mathbf{K}+q}^{*}(\mathbf{r}) \exp [i(q+\mathbf{K}) \mathbf{r}] \psi_{n \mathbf{k}}(\mathbf{r}) d^{3} r
\end{aligned}
$$

where $V_{c}$ is the volume of the primitive unit cell. To carry out the integration, for each $1 / 48$ th of the first Brillouin zone there are ten $\mathbf{K}$ points taken into account. If the vector $(\mathbf{K}+\mathbf{q})$ lies outside the Brillouin zone it can be brought back into it by the addition of a suitable reciprocal lattice vector $\mathbf{Q}^{*}$. 
The Fermi-Dirac distribution funtermational tournal of Scienseand Researth (IJSR)

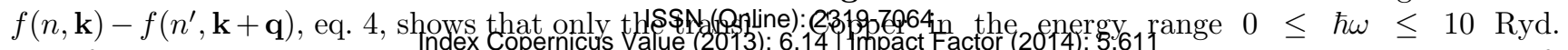

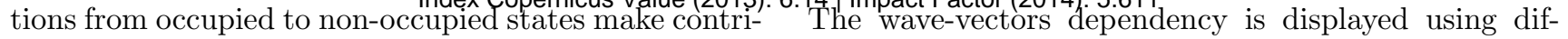
butions to the dielectric function. The summation over the non-occupied bands is restricted in the calculation of the imaginary part, $\epsilon^{\prime \prime}$, only to those bands whose energies are lying with a maximum value $\hbar \omega$ above the Fermi energy. While the summation in the calculation of the real part, $\epsilon^{\prime}$, is not restricted.

The augmented-plane-wave method (APW) which was first proposed by Slater $[11,12]$ is used frequently to calculate the energy bands of metals $[13,14]$. The basic idea behind the APW is that the potential in a spherical region centered at each atom is spherically symmetric, hence, the wave-functions are rapidly varying and exhibit atomic-like solution. On the other hand, the potential in the interstitial regions is flat, consequently, it provides plane waves. The augmented plane waves are constructed by matching solutions of the Schrdinger equation within each sphere with plane-wave solutions in the interstitial region. Although the APW is an efficient method for the computation of the bands, it is worth reporting that the APW is not completely rigorous. The accuracy of the findings depends on some adjustable parameters such as the radius of the spherically symmetric region, the spherically symmetric potential, and the constant potential in the interstitial region. Moreover, it is only applicable to an unphysical spherical muffin-tin potential. Therefore, in our calculations, the Bloch state wave function $\psi_{n \mathbf{K}}$ are calculated employing the modified augmented-plane-wave method (MAPW) [15, 16] with the Chodorow potential [17]. Different reasons attribute to that: The MAPW itself is applicable to spherical and non-spherical potentials, the calculated wave-functions are orthogonal to the functions of the electron core electrons, the calculated wavefunctions and their first derivatives are continuous, and finally the modified APW converges with the rapidity of the usual APW. Furthermore, the Chodorow potential [17] yields band energies in agreement with the experiment $[18,19]$. For the present work, every Bloch function is approximated by about 60 plane waves outside the inscribed APW-sphere and 9 spherical waves inside it. The corresponding eigenvalue problem of the rank 70 means that for every k-point there are 70 different eigenstates, 10 below the Fermi level and 60 above it.

It is well known that the measurements of the wave-vector and frequency-dependent complex dielectric function $\epsilon(\mathbf{q}, \omega)$ for real metals is tedious. Therefore, the results of our calculations will be compared with the analytical dielectric function (Lindhard function [20]) for free electron gas.

\section{Results and discussion}

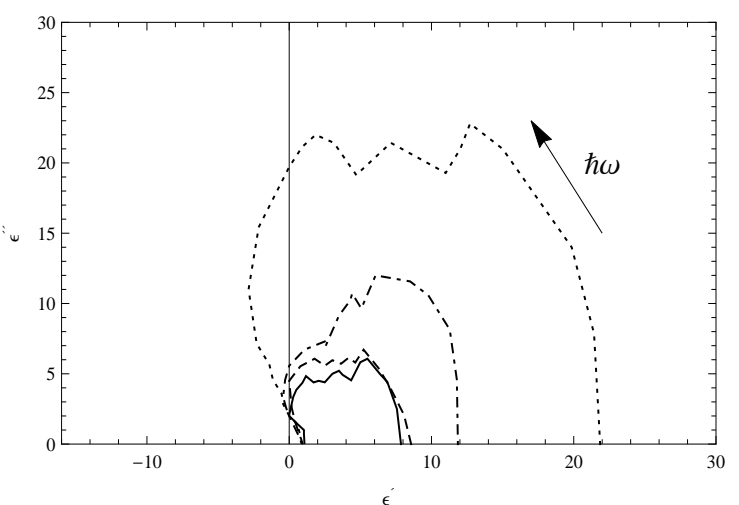

ferent line styles: Solid $0.52 \pi / a$, Dashed $0.43302 \pi / a$, Dotted-Dashed $0.35352 \pi / a$, and Dotted $0.252 \pi / a$

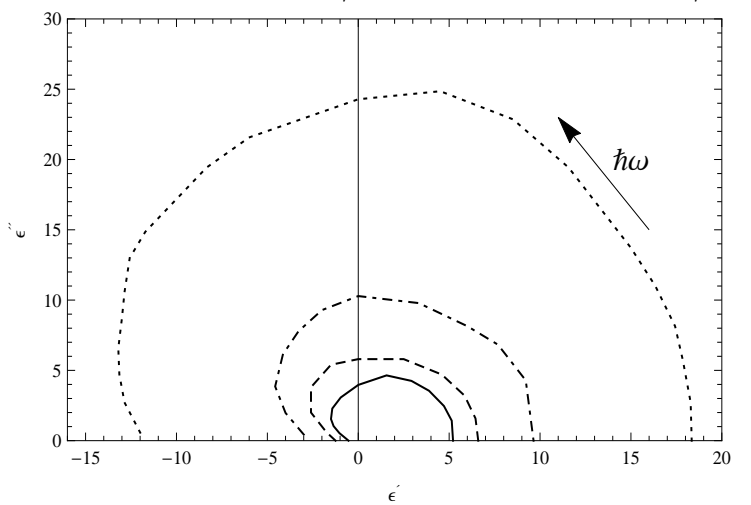

Figure 2: The wave-vector Cole-Cole diagram of the free electron gas in the energy range $0 \leq \hbar \omega \leq 10 \mathrm{Ryd}$. The wave-vectors dependency is displayed using different line styles: Solid $0.52 \pi / a$, Dashed $0.43302 \pi / a$, Dotted-Dashed $0.35352 \pi / a$, and Dotted $0.252 \pi / a$.

The Cole-Cole diagram of the dielectric complex function of Copper and free electron gas are displayed in figures (1) and (2), respectively. Discrepancies are expected. The dielectric function of the free electron gas is determined by the collective oscillation resulting from the long range effects of Coulomb interaction. The dielectric function of the electron gas in real metals is modified by the polarization of the core electrons and the inter-band transition even if the local field effects are neglected. Unlike the dielectric behavior of the free electron gas, the real part of the copper dielectric function exhibits two longitudinally polarized modes (i.e., $\left.\epsilon^{\prime}(\mathbf{q}, \omega)=0\right)$ instead of one with respect to the frequency, namely $\epsilon^{\prime}\left(\mathbf{q}, \omega_{1}\right)$ and $\epsilon^{\prime}\left(\mathbf{q}, \omega_{2}\right)$, where $\omega_{2}>\omega_{1}$. The dependence of both frequencies, $\omega_{1}$ and $\omega_{2}$, on the wave-vector is quantified in table (1). The two modes almost merge at $q=0.52 \pi a^{-1}$ and no longitudinal modes exist ( i.e, $\epsilon^{\prime}(\mathbf{q}, \omega)>0$ ) for all $q>0.52 \pi a^{-1}$.

Table 1: The frequencies at which the real dielectric component of Copper is vanished as a function of the wave-vector. Note: $\omega_{2}>\omega_{1}$.

\begin{tabular}{|c|c|c|}
\hline$q /\left(2 \pi a^{-1}\right)$ & $\omega_{1} \quad(\mathrm{Ryd})$ & $\omega_{2} \quad(\mathrm{Ryd})$ \\
\hline$\left(\frac{1}{4}, 0,0\right)$ & 0.28 & 0.72 \\
\hline$\left(\frac{1}{4}, \frac{1}{4}, 0\right)$ & 0.53 & 0.75 \\
\hline$\left(\frac{1}{4}, \frac{1}{4}, \frac{1}{4}\right)$ & 0.59 & 0.63 \\
\hline$\left(\frac{1}{2}, 0,0\right)$ & 0.85 & 0.86 \\
\hline
\end{tabular}

Table 2: The imaginary dielectric component $\epsilon^{\prime \prime}(\mathbf{q}, \omega)$ of Copper at the frequencies $\omega_{1}$ and $\omega_{2}$.

\begin{tabular}{|c|c|c|}
\hline$q /\left(2 \pi a^{-1}\right)$ & $\epsilon^{\prime \prime}\left(\mathbf{q}, \omega_{1}\right)$ & $\epsilon^{\prime \prime}\left(\mathbf{q}, \omega_{2}\right)$ \\
\hline$\left(\frac{1}{4}, 0,0\right)$ & 19.38 & 1.95 \\
$\left(\frac{1}{4}, \frac{1}{4}, 0\right)$ & 5.48 & 2.06 \\
$\left(\frac{1}{4}, \frac{1}{4}, \frac{1}{4}\right)$ & 4.39 & 3.97 \\
$\left(\frac{1}{2}, 0,0\right)$ & 1.95 & 1.92 \\
\hline
\end{tabular}

Table (2) gives the imaginary dielectric components when the real component is vanished. $\epsilon^{\prime \prime}\left(\mathbf{q}, \omega_{1}\right)$ decreases almost quadratically via increasing the wavevector, it is small but not negligible. Therefore, very sharp resonance peaks are not expected to be found in the $\mathrm{Cu}$ energy loss spectrum [10]. Moreover, as can be seen in figures (1) and (2), the static dielectric function - which is important in determining the static screening of electric fields - is a pure real quantity and decays sharply with increasing the wave-vector. One of the 
interesting features of the Copper Internatienal Journal of Scien€ and Besearch.(IJSR) T.D., Lee T.C., Badami D., 2004

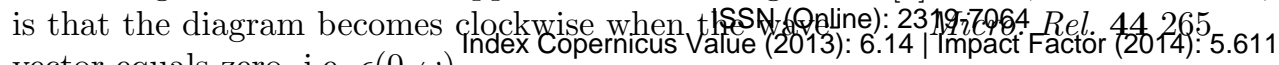

vector equals zero, i.e, $\epsilon(0, \omega)$.

\section{Conclusion}

The wave-vector Cole-Cole diagram of Copper as well as free electron gas has been calculated. The static dielectric functions $\epsilon(\mathbf{q}, 0)$, the shape of $\epsilon^{\prime \prime}(\mathbf{q}, \omega)$ and the zeros of $\epsilon^{\prime}(\mathbf{q}, \omega)$ have been found to be wave-vector functions. The free electron gas exhibits one plasmon, while the electron gas in Copper metal exhibits two plasmons for wave-vectors smaller than $0.52 \pi a^{-1}$. The second author thanks W.-D. Kraeft and S. Saafan for helpful discussions.

\section{References}

[1] Nalwa H.S., 1999 Handbook of low and high dielectric constant materials and their applications, Academic press, USA \& UK

[2] Kittel C., 2005 Introduction to solid state physics, Jon Wiley \& Sons Inc

[3] Debye P., 1929 Polar Molecules Chemical Catalog, New York.

[4] Cole K.S. and Cole R.H., 1941 J. Chemical Physics 9341

[5] Gupta T., 2009 Copper Interconnect Technology, Springer
[7] Maex K., Baklanov M.R.,Shamiryan D., lacopi F., Brongersma S.H., and Yanovitskaya Z.S., 2003 J. Appl. Phys. 938793

[8] Havemann R.H., Hutchby J.A., 2001 Proceedings of the IEEE $\mathbf{8 9} 586$

[9] Adler S., 1962 Phys. Rev. 126413

[10] Kubo Y., Wakoh S., and Yamashita J., 1976 J. Phys. Soc. Japan 411556

[11] Slater J.C., 1937 Phys. Rev. 51846

[12] Slater J.C., 1953 Phys. Rev. 92603

[13] Harrison W.A., 1970 Solid state theory Tata McGraw-Hill, Inc. New York

[14] Kleinman L. and Shurtleff R., 1969 Phy. Rev. 188 1111

[15] Bross H. and Stohr H., 1964 Phys. Lett. 825

[16] Bross H., 1968 Z. Phys. 215485

[17] Chodorow M.I., Dissirtation, MIT, 1939

[18] Segal B., 1962 Phys. Rev. 125129

[19] Burdick G.A., 1963 Phys. Rev. 129138

[20] Lindhard J., 1954 Mat.-fys. Medd. 288 